\title{
Application of GPS to Train Control Systems for Secondary Lines
}

\begin{abstract}
Haruo YAMAMOTO
Senior Researcher, Laboratory Head,

Hiroyuki SUGAHARA

Assistant Senior Researcher,

Train Control Systems Laboratory, Signalling and Telecommunications Technology Division

With a view to reducing the cost of on board positioning and speed detection equipment in train control systems using radio communications, for secondary lines, GPS positioning performance was investigated in the case where it is used in combination with MSAS (MTSAT Satellite-based Augmentation System). Results showed that it was possible when using this system to locate the vehicle on the track by using horizontal protection level, when on open track sections. Furthermore, tests carried out using only on board equipment show that it should be possible to build a system which does not rely on any ground installations.
\end{abstract}

Keywords: secondary lines, train control, radio communications, on board location detection, GPS, MSAS

\section{Introduction}

A low cost train control system to improve secondary line management is currently being developed. In order produce an inexpensive train control system for secondary lines which does not rely entirely on ground equipment by using radio transmission technology, it was necessary to check the applicability of general purpose radio transmission technology to the achieving the abovementioned objectives as well as on board positioning. Trackside to on board transmission of information would only then be used in the case of specific track features such as stations and level crossings. Figure 1 is an image of what this train control system would look like.

The on board system in question would provide continuous positioning and speed control. A tachometer generator, a sensor with proven performance and which is generally used as safety equipment, would be used to carry out these functions. However, in the case of short train consists which often operate on secondary lines, any weakening of the brake force on an axle to which the tachometer generator is attached is problematic, given the significant influence this has on the overall braking force of the consist. Furthermore in some cases it may not be possible to attach a tachometer generator to axles other than driving axles. Even if it were possible to attach them to two axles, the probability then of slipping and sliding occurring on them at the same time would be greater than on main lines. Consequently, the tachometer would have to be used in conjunction with a location and speed detection function which does not rely on driving axle spin.

GPS is one possible solution. Since secondary lines are often in an unobstructed environment and are open track, except in mountainous areas, it is easy to use GPS. Then, study on making GPS possess location and velocity detection function equivalent to the tachometer generator for safety equipment was performed. This report describes the results obtained.

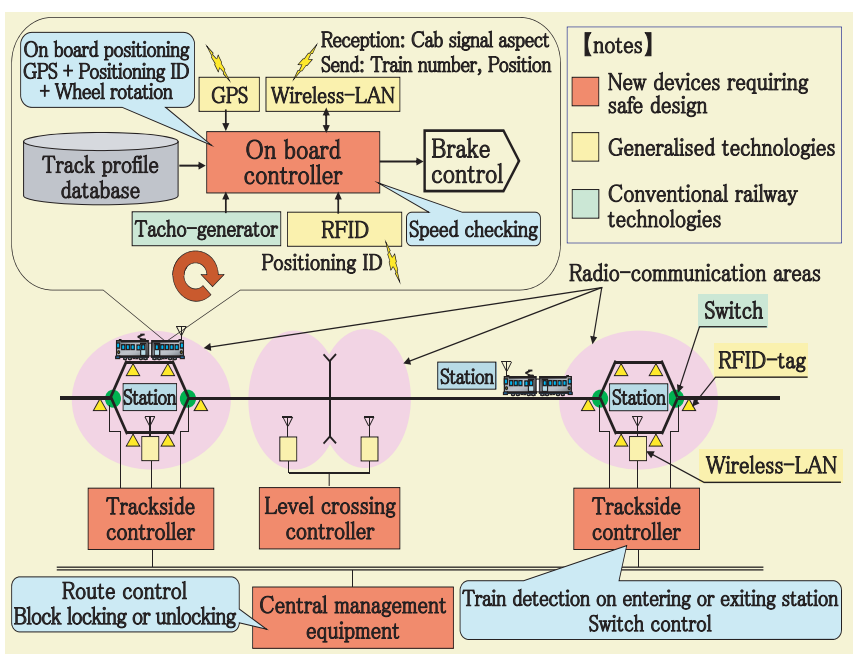

Fig. 1 Image of train control system using radio
communications for secondary lines

\section{About MSAS}

Satellite navigation systems of which GPS is a well known example, are recognised as being an important part of social infrastructure. The U.S. Government has officially announced that GPS would from now on be open to the whole world. In addition, since Japan's joint statement with the US on the 5 September 1998 on GPS, Japan actively cooperates for the promotion of GPS as a single worldwide standard.

Japan is the No.1 GPS user country in the world. The Japan Coast Guard and the Civil Aviation Bureau, the Ministry of Land, Infrastructure, Transport and Tourism, employ GPS international standard augmentation systems for navigation support. Augmentation systems raise positioning accuracy and reliability in cases where simple GPS performance is insufficient.

The Civil Aviation Bureau uses the augmentation system MSAS (MTSAT Satellite-based Augmentation System) in order to employ GPS as a navigation tool in civil 
aviation. GPS positioning reliability can be enhanced by receiving GPS signals together with MSAS signals via a SBAS (Satellite-Based Augmentation System) receiver (Fig. 2). Since MSAS signals have the same frequency as GPS signals, they can be received by GPS antenna.

Jointly used with MSAS, errors such as malfunction data, orbital errors, clock errors, and ionosphere timedelay errors on GPS can be corrected. Horizontal positioning errors for example are reduced to less than several meters, and information inside the error-range (protection level) is guaranteed to within a probability of 99.99999\%. Furthermore MTSAT (Multi-functional Transport Satellite) which broadcasts MSAS signals can be used for satellite positioning as an equivalent to GPS. As geostationary satellites, MTSATs have relatively low elevation: for example 48 degrees in Tokyo and unlike GPS Satellites, they offer permanent domestic coverage. MTSAT is a two-set organisation, and since it is under the strict control of two aeronautical satellite centres situated in two domestic locations, the reliability of the system is high. In addition, there is no usage fee.

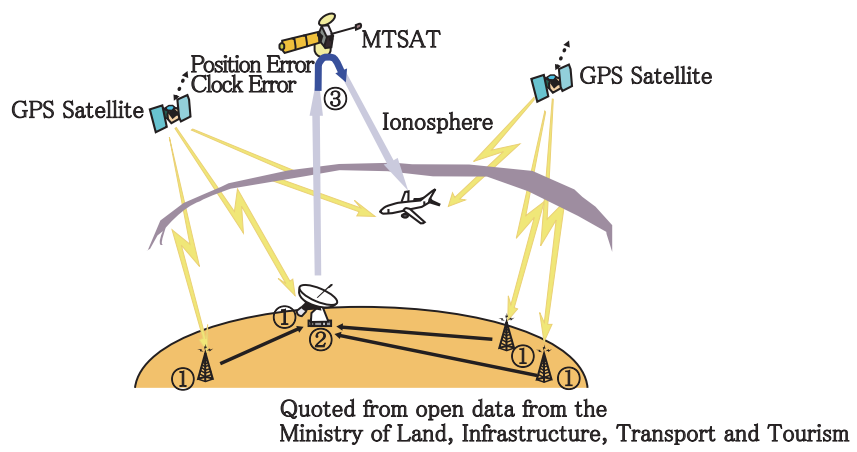

Fig. 2 MSAS

\section{GPS positioning performance in the case of joint use with MSAS}

In order find a low cost, reliable on board location and speed detection function applicable to train control systems for secondary lines, the applicability of GPS and MSAS was examined.

However, MSAS is an aviation-oriented system, and as one can imagine, not a system originally meant to be used with trains. In a railway environment, GPS signals may be severely influenced by interception, reflection, etc. due to topography or buildings along the line, or be simply undetectable, for example in tunnels. Therefore, to verify GPS positioning performance when used together with MSAS, measurements were made at a fixed point under open sky, and on a train respectively, and positioning performance was evaluated on the basis of these measurement results.

\subsection{Positioning performance at a fixed point under open sky}

In order to confirm the effectiveness of MSAS, GPS positioning was measured over a long period of time at a fixed point under open sky. Measurements were taken over half a year from September 2007, when MSAS started operation. As an example of the outcome of this a distribution of the positioning results measured every second as part of a continuous measurement over seven days is shown in Fig. 3. In locations where GPS and MSAS signal reception was good, positioning errors are reduced to about $2 \mathrm{~m}$.

On the other hand, HPL (Horizontal Protection Level) showed variations in general of between $14 \mathrm{~m}$ to $100 \mathrm{~m}$, depending on certain changeable conditions such as satellite constellation. An example of the variation in status over 24 hours during the measurement period of Fig. 3 is shown in Fig. 4.

When integrity cannot be maintained due to anomalies, HPL expands. At the same time, these anomalies can be detected from the information output. Similarly, when MTSAT is undergoing maintenance such as orbital adjustment etc., this is detected as an anomaly. In such situations, an alternative system may be used, as mentioned above.

HPL covers the positioning error more than enough when reception conditions are good, and so there are plans to improve the HPL value by narrowing it down [1].

\subsection{On board positioning performance}

\section{2. 1 Test method}

Tests were carried out on Railway Technical Research Institute test tracks with rolling stock in order to identify location and speed detection errors from GPS positioning when used together with MSAS on a moving train. Eight RFID tags with a static response range of about $0.5 \mathrm{~m}$ were arranged along the section of track used in the running tests measuring approximately $620 \mathrm{~m}$. While recording SBAS receiver data emitted every 0.2 seconds on the moving train, information received from the RFID tags and pulses from the tachometer generator

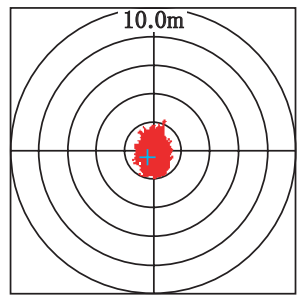

Receiving MSAS signal (PRN129)

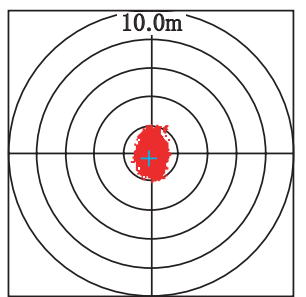

Receiving MSAS signal (PRN137)

Fig. 3 Example of seven-day continuous positioning results in a location where reception conditions are good 

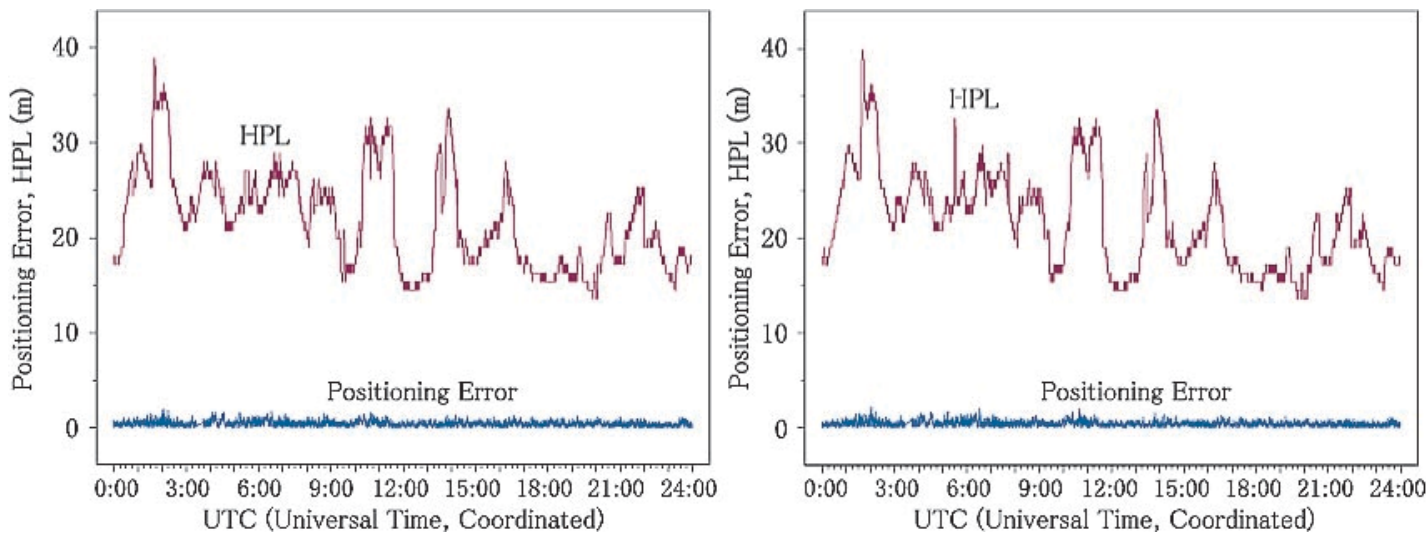

Fig. 4 Example (left: PRN129, right:PRN137) of HPL variation by location where reception conditions are good

attached to the non-driving shaft were recorded together according to a time-keeping device corrected by the GPS receiver 1PPS every minute (Fig. 5). The rolling stock was run 184 times for the tests, and 93,113 pieces of data were collected. The vehicle location and speed whilst in motion were determined for each time series on the basis of output results from the eight RFID tags and the track centre line; from this it was possible to evaluate the accuracy of location and speed information etc. collected from the SBAS receiver.

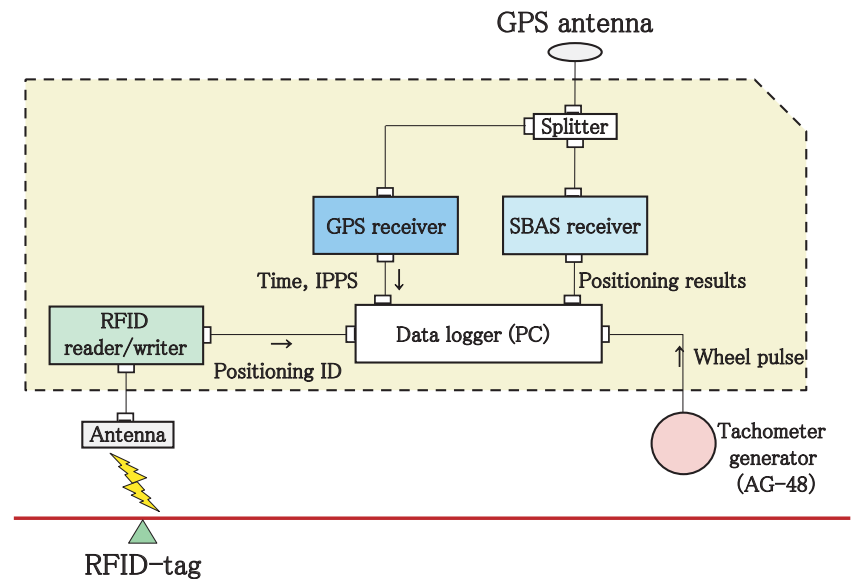

Fig. 5 Configuration of measuring system

\section{2. 2 Test results}

(1) Positioning errors and HPL

An example of test results is shown in Figs. 6 and 7. Buildings, for the purpose of the experiment were randomly place around a test track. In particular trees were located close to the departure end of the test running track, to reduce the quality of reception conditions for GPS signals. In such locations, positioning error increased and HPL expanded noticeably.

The relationship between positioning errors and HPL for the complete set of data from these tests when MSAS is normal is shown in Fig. 8. The figure includes many positioning results for cases where there were few acquisition satellites and the satellite constellation was poor, due to the train starting to run immediately after powering up and before the SBAS receiver could begin positioning etc., as well positioning results in locations around the test track where reception conditions were not good (only the positioning results from three GPS Satellites and MTSAT are included). Therefore, although the HPL while the train was running recorded a maximum of $526 \mathrm{~m}$ and the positioning error recorded a maximum of $41 \mathrm{~m}$, the positioning error did not exceed the HPL.

(2) Ellipsoidal height, HDOP (Horizontal Dilution of Precision)

Variation in ellipsoidal height and HDOP observed during test runs and analysed in Figs. 6 and 7 is shown in Fig. 9. HDOP is a module which shows the degree of deterioration in presumed accuracy from the satellite constellation used for the positioning, and the larger numeri-

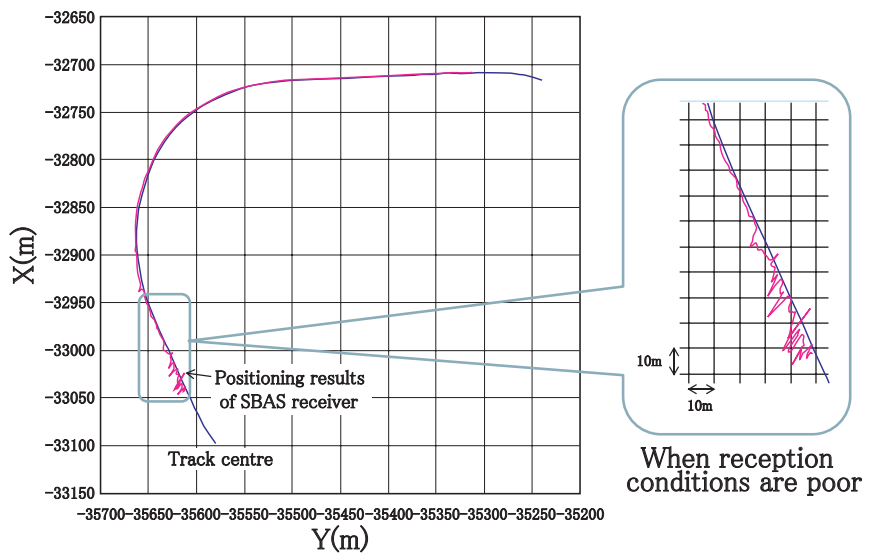

Fig. 6 Example of positioning results in case of test track running (plane rectangular coordinate)

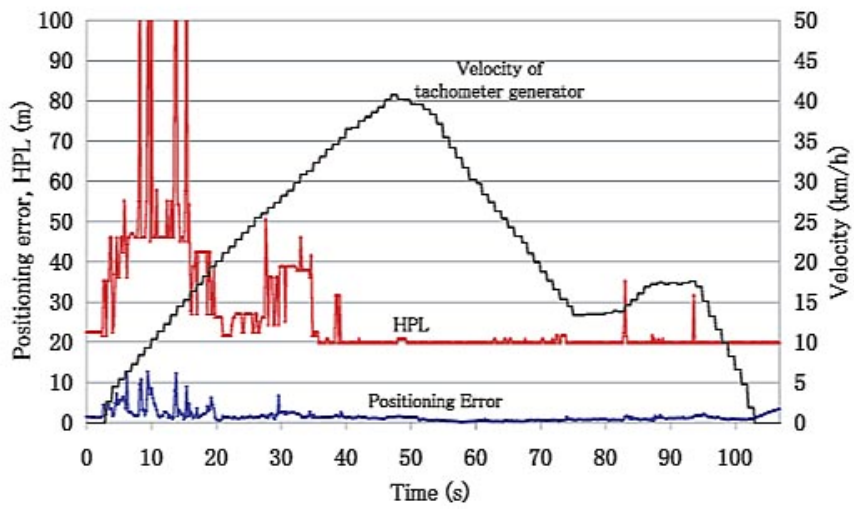

Fig. 7 Example of positioning results in case of test track running (positioning error, HPL) 

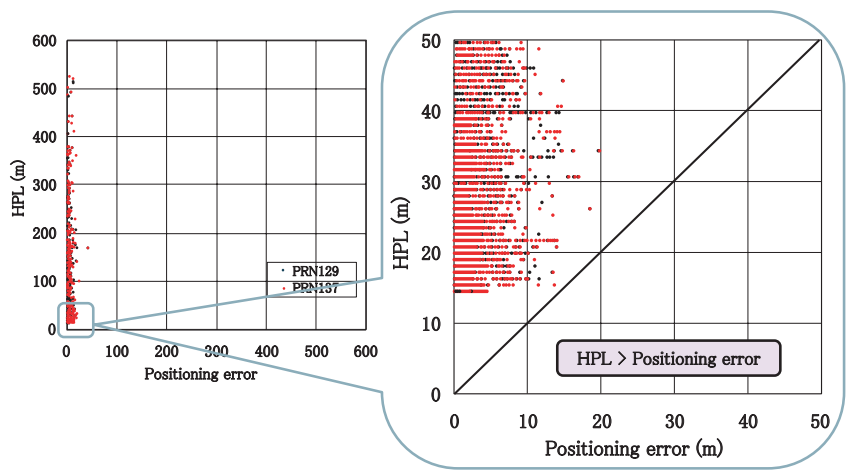

Fig. 8 MSAS HPL performance during running on test track

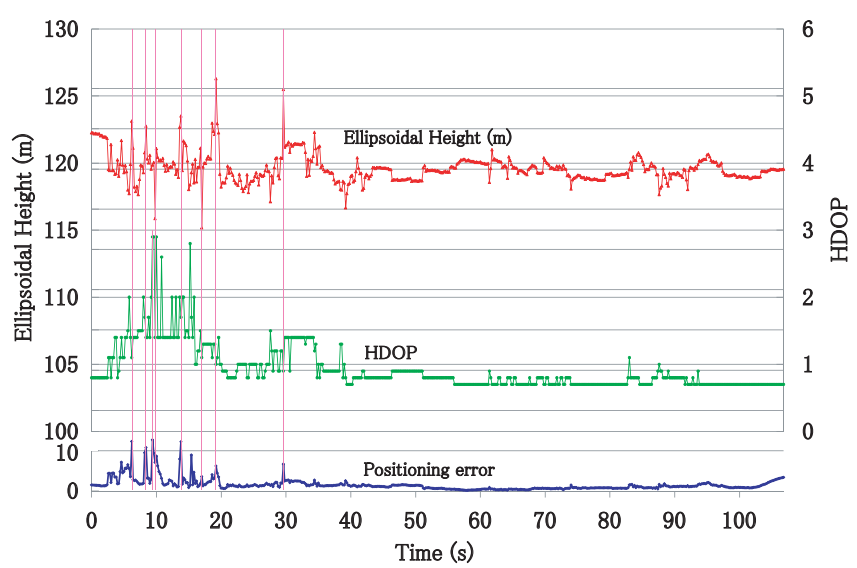

Fig. 9 Example of positioning results in case of running on test track (ellipsoidal height, HDOP)

cal value, the poorer the accuracy. When horizontal positioning errors increased, there was a tendency for variation in the height or HDOP to grow. Therefore, it may be possible to estimate deterioration in positioning accuracy from these absolute values.

(3) Ground speed

The ground speed in the running tests analysed in Figs. 6 and 7 is shown in Fig. 10. The passing speed obtained from the positioning coordinates emitted every 0.2 second is shown together with the ground speed in Fig. 11. Fig. 12 represents Fig. 11 adapted to show error plotted against base speed.

From these figures, it turns out that even in locations where reception is poor such that there are positioning errors as mentioned above, the HPL and HDOP increase, and ellipsoid quantity changes, the ground speed is comparatively stable, and its accuracy is very good compared with the passing speed converted from the positioning coordinates. Ground speed is calculated from the Doppler effect of the GPS signal carrier accompanying reception side movement. Although multipath also influences carrier frequencies, the impact is negligible since it is very small compared with the pseudo-range.

As mentioned above, when reception conditions are poor, it is thought that dead-reckoning based on movement distance obtained by integrating the speed is effective for short time-spans.

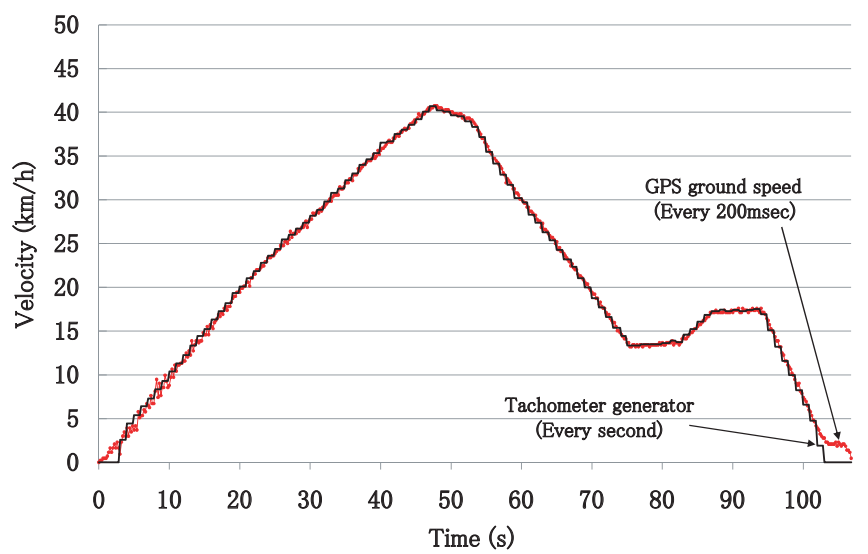

Fig. 10 Example of positioning results in case of running on test track (ground speed)

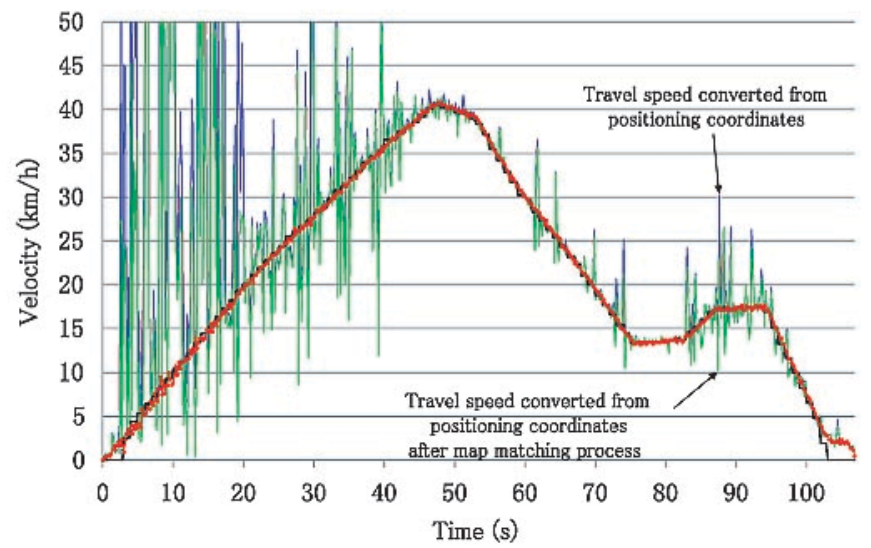

Fig. 11 Example of positioning results in case of running on test track (ground speed, travel speed converted from positioning coordinates)

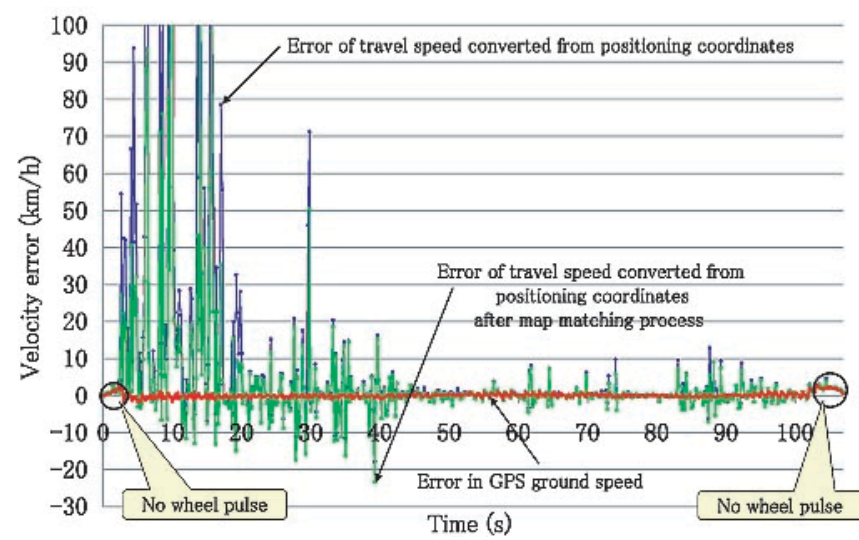

Fig. 12 Example of positioning results in case of running on test track (Error of the ground speed and the travel speed converted from positioning coordinates against base speed)

\section{(4) Direction}

The direction of test runs from Figs. 6 and 7 is shown in Fig. 13. Although direction is acquired together with ground speed information and is comparatively stable during test runs, deviation becomes very noticeable at $5 \mathrm{~km} / \mathrm{h}$ or less, and becomes undefined while the train is stopped. 


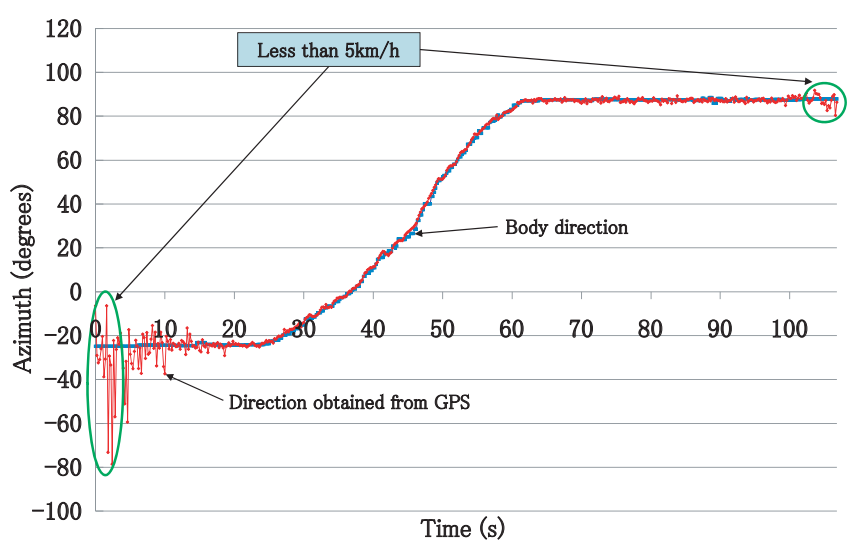

Fig. 13 Example of positioning results in case of test track running (direction)

\section{2. 3 Summary of the test results}

The summary of results from the test runs is shown in Table 1.

\section{Study on the application of GPS and MSAS to on board location detection}

Although limited to the test range, the MSAS HPL includes positioning errors as described in the preceding chapter. Therefore, it may be possible to use GPS positioning results and HPL together with MSAS for train detection information. Studies were therefore carried out to find a method to obtain on board location and speed detection functions for the train control system on secondary lines by applying both GPS and MSAS.

\subsection{Functional requirement}

The requirements in terms of accuracy of train location detection include ability to distinguish the track on which a train is located even when there are several tracks, and positioning of the train to within several or less metres. In terms of reliability it must be possible to locate a train continuously and for safety reasons, the dangerous failure rate must be below $10^{-9} / \mathrm{h}$ to avoid collision and derailment of a train due to incorrect positioning information. With regard to permissible errors, some easing is possible depending on the demands for location detection on the specific lines where the system is to be applied.

\subsection{The safety guarantee requirement}

MSAS is not the same system as GPS. Although pseudo-range error and failures during transmission radio waves from a satellite to the ground are common to both systems. Also, they are detected by MSAS ground installations and the user can receive them through MSAS augmentation information. However, since GPS signals and MSAS signals are received through a single receiver by the user receiver failures need to be handled as a common cause failure.

MSAS functions including PL (Protection Level) have been tried and tested for ensuring aviation safety. Once it is understood how MSAS works, the fact that it meets safety requirements for use in aviation and that it guarantees air traffic safety, should serve as evidence to show that it can be used in other similar demanding contexts. On this basis, were MSAS to be applied to a train safety control system, certain factors would have to be examined: ensuring that requirements in terms of accuracy and safety for the train safety control system are higher than

Table 1 Summary of test results on test track

\begin{tabular}{|c|c|c|}
\hline Output information & Test result & Notes \\
\hline Latitude and Longitude & $\begin{array}{l}\text { If receiving conditions are good, positioning errors } \\
\text { (horizontal) will reduce to about } 2 \mathrm{~m} \text {. }\end{array}$ & $\begin{array}{l}\text { If judging by the quality of accuracy, applicable to } \\
\text { locating the position of the vehicle on the track }\end{array}$ \\
\hline HPL & $\begin{array}{l}\text { - More than } 14 \mathrm{~m} \text { and varies in the range up to } 100 \mathrm{~m} \text { in general } \\
\text { - Expands conspicuously as positioning error increases when } \\
\text { reception conditions are poor } \\
\text { - Included an actual positioning error (in the test range) }\end{array}$ & Availability for area occupied by train information \\
\hline $\begin{array}{l}\text { Ellipsoidal height } \\
\text { (Height information) }\end{array}$ & - Tendency to have larger variation as positioning error increases & $\begin{array}{l}\text { It is possible to guess whether positioning error is } \\
\text { large or not by number of variations }\end{array}$ \\
\hline HDOP & - Tendency to become larger as positioning error increases & $\begin{array}{l}\text { It is possible to guess whether positioning error is } \\
\text { large on not by the value }\end{array}$ \\
\hline Ground speed & - Comparatively stable even where reception conditions are poor & $\begin{array}{l}\text { It is effective for dead-reckoning for short time spans } \\
\text { when positioning error is large. }\end{array}$ \\
\hline Direction & $\begin{array}{l}\text { - Comparatively stable even where reception conditions are poor } \\
\text { - At } 5 \mathrm{~km} / \mathrm{h} \text { or less, variation stands out; becomes undefined when } \\
\text { stopped }\end{array}$ & Available for verification of ground speed \\
\hline
\end{tabular}


in aviation safety systems, and taking into account the fact that reception conditions will be worse along railway tracks than in the open sky.

In order to tackle situations where lack in accuracy of information makes it difficult to distinguish between lines, it is maybe necessary to use information about turnout direction etc. as a remedy to identify the track. To this end it would be necessary to build a mechanism to ensure detection of exact initial positions. Furthermore to meet the high safety requirements, it would be necessary to examine mechanisms which guarantee positioning results using alternative means, such as line information, sensors, etc. As for the reception environment, studies would probably have to be carried out on a judgment logic for selecting the position results received under good conditions, and to make up lower parts of the positioning frequency rate with a tachometer generator etc. If the above three points are solved, creating an on board location detection function for the train control system using MSAS would be possible.

\subsection{Method for detecting location and speed}

\section{3. 1 Instrument configuration}

The location and the speed detection function of the CARAT (Computer And Radio Aided Train control system) basically relies on two or more tachometer generators and a geographical-point detection device, and uses inert sensors and GPS accessorily, depending on location detection requirements [2]. However, since the location and speed detection function currently being studied is for secondary lines, there is a strong pressure to reduce cost. Therefore, the geographical-point detection device for location correction shown in Fig. 1 is not included in the basic configuration, and is only added when location detection accuracy demands it in particular. In addition, rolling stock was not equipped especially with tachometer generators as safety devices; instead it was decided to make use of the already installed as speedometers. According to the description above, there is no fundamental need for ground installations and rolling stock only needs to be equipped with a GPS antenna on the roof in addition to the controller. In this way, installation costs are kept to a minimum and maintenance costs are also reduced.

\section{3. 2 Basic methodology}

The block diagram describing location and speed detection is shown in Fig. 14.

The method is based on the following three points:

(1) Velocity detection and distance integration based on a tachometer generator

(2) Velocity and distance correction in the case of slip and skid based on back-and-forth acceleration measured by individual-joint-acceleration meters

(3) Absolute location detection (location correction, initial-position detection) based on GPS when used together with MSAS, and calibration of speed The core instrument for location and speed detection is one tachometer generator, which is reinforced by

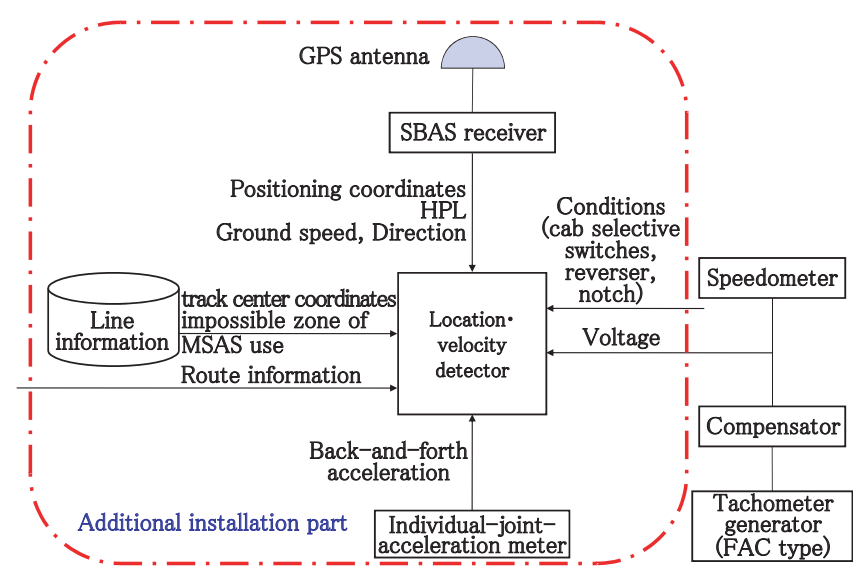

Fig. 14 Location and speed detection block diagram

an individual-joint-acceleration meter which is not influenced by slip and skid. The output of the established tachometer generator used as a speedometer has been adjusted so that speed indication errors are within the permissible value margin of $\pm 2-3 \mathrm{~km} / \mathrm{h}$. However, in the case of a voltage output tachometer generator distance integration is less accurate than pulse counts. Therefore, while using the ground speed obtained from GPS when the train is running for calibration of speed information based on the tachometer generator, frequent corrections are made to location on the basis of GPS positioning results when used together with MSAS along sections under open sky. For sections with obstacles, such as tunnels, where GPS and MSAS cannot be used, the corrected speed based on the tachometer generator supported by the individual-joint-acceleration meter serves as back up. Locations on a line where GPS and MSAS are available can be determined beforehand.

\section{3. 3 Line information}

As is required in CARAT, in order to express the track layout required for train location detection, information such as track block sections which are formed by dividing the track by turnout / confluence, clearance limits, block length and interconnection of blocks, etc. should be provided. Furthermore, in order to correct the backand-forth acceleration measured with an individualjoint-acceleration meter, the magnitude and location of gradients identified in relation to specific track blocks are added, and in order to apply GPS positioning, track centre coordinate strings are also identified and added in relation track block sections (Fig. 15). Although track centre coordinates should include at least latitude and longitude, where possible altitude should also be also included.

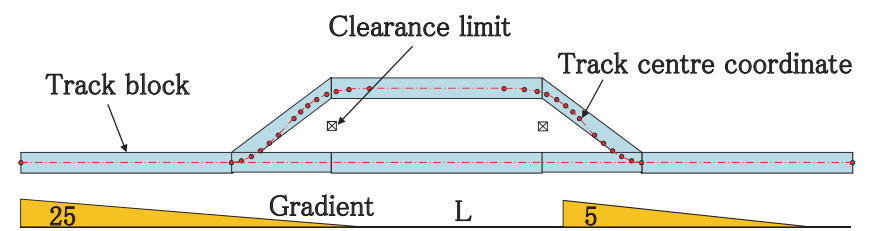

Fig. 15 Concept of line information 


\section{3. 4 Detection of train location}

(1) Locations under open sky

In this method, the area occupied by a train is determined by combining the train length, data about position coordinates and the HPL obtained from the SBAS receiver and the track centre coordinate string (Fig. 16). If an error in positioning coordinates causes the occupied area to shift sideways off the track, this is of course irrational, however, the HPL should expand as the positioning error increases. Nonetheless as described in Section 3.2, in the case areas not under open sky, HPL expands to a conspicuous level as positioning error increases. Under such circumstances, HPL can no longer offer the location correction function. Consequently HPL should only be used for location correction when it satisfies accuracy requirements for train location detection. As such the suitability of positioning results is checked on the basis of the specificities in railway vehicle movement (running on continuous tracks, vertical variation is small) and the ground speed and direction information which can be obtained from the Doppler effect. By performing location correction only in cases where positioning error is assumed to be small, this helps to avoid a phenomenon whereby train position errors are magnified. Although frequency of positioning falls by $17 \%$ as a result of squeezing data from tests using rolling stock as described in Section 3.2 into the relevant conditions based on the specificity described in Section 3.2.2, the maximum value of the error in track longitudinal direction decreases to $2.0 \mathrm{~m}$ or less which is equivalent to the fixed point (Table 2). Given current MSAS performance, it is generally understood that it is difficult for HPL to be maintained at $20 \mathrm{~m}$ or less, however, there is hope for promising results on the basis of the stated plans to improve this MSAS performance.

In addition, in order to calibrate detected speed according to the tachometer generator used as a speedometer with a ground speed obtained from the GPS, the relationship between the tachometer generator output dur-

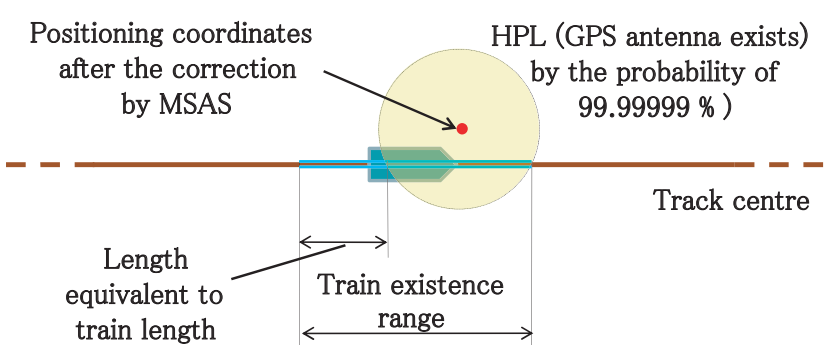

Fig. 16 Method for detecting area occupied by a train

ing coasting and the ground speed from GPS is memorised and stored.

(2) Location under closed sky

Dead-reckoning from the last train location is performed on the basis of speed measured by the tachometer generator backed up by the individual-joint-acceleration meter. After booting up the equipment, speed is obtained using the conversion rate from the input voltage to the speed of the speedometer until the abovementioned calibration information (relationship between the tachometer generator output and the ground speed from GPS) is stored and finalised. The speed error in this case is estimated at $\pm 2-3 \mathrm{~km} / \mathrm{h}$, and accuracy increases further after the calibration information is defined. The area occupied by the train determined through dead-reckoning guarantees safety against speed related errors. This is done by taking into account errors stored while running and adding the length expanded proportionally to the running distance covered from the last correction implementation location to the area occupied by the train and determined at the correction location (Fig. 17).

Zones where MSAS cannot be used due to satellites not being visible should be predefined. While monitoring through dead-reckoning is in operation to check presence of the train in the zone, cases where positioning coordinates and HPL are not available in a zone where MSAS can be used, are regarded as equipment failures.

Table 2 Verification conditions for train location detection and effect of reducing the maximum error

\begin{tabular}{|c|c|c|c|c|}
\hline No. & $\begin{array}{l}\text { Classification of the } \\
\text { verification method to be added }\end{array}$ & Verification conditions & $\begin{array}{l}\text { Maximum error } \\
\text { in the track longi- } \\
\text { tudinal direction }\end{array}$ & $\begin{array}{l}\text { Positioning } \\
\text { frequency }\end{array}$ \\
\hline & & SBAS integrity (Positioning integrity mode) & $39.9 \mathrm{~m}$ & Basis \\
\hline 1) & Output information from receiver & $\mathrm{HPL}<80 \mathrm{~m}^{* 1)}$, HDOP $<2$ & $14.5 \mathrm{~m}$ & $-2 \%$ \\
\hline 2) & $\begin{array}{l}\text { Specificity of movement of railway } \\
\text { vehicle }\end{array}$ & $\begin{array}{l}\text { Following conditions are added to } 1): \text { Height varia- } \\
\text { tion }<3 \mathrm{~m} \text {, Amount of compensations in the track } \\
\text { transverse direction in the case of map matching }<\end{array}$ & $2.0 \mathrm{~m}$ & $-7 \%$ \\
\hline 3) & $\begin{array}{l}\text { Direction information obtained } \\
\text { from GPS }\end{array}$ & $\begin{array}{l}2 \mathrm{~m} \text { Fowing conditions are added to } 2): \text { Direction } \\
\text { variation }< \pm 15 \text { degree }\end{array}$ & $2.0 \mathrm{~m}$ & $-10 \%$ \\
\hline 4) & Ground speed obtained from GPS & $\begin{array}{l}\text { Following conditions are added to } 3 \text { ) : Movement } \\
\text { length after map matching is within the limits of the } \\
\text { assumed error (less than } \pm 5 \mathrm{~km} / \mathrm{h}^{* 2)} \text { ) of ground speed }\end{array}$ & $2.0 \mathrm{~m}$ & $-17 \%$ \\
\hline
\end{tabular}

*1) HPL is set at $80 \mathrm{~m}$ or less because the present MSAS has large variation even under normal conditions

*2) Since speed error is less than $\pm 5 \mathrm{~km} / \mathrm{h}$ for conditions listed under 3 ) (based on the test result) 


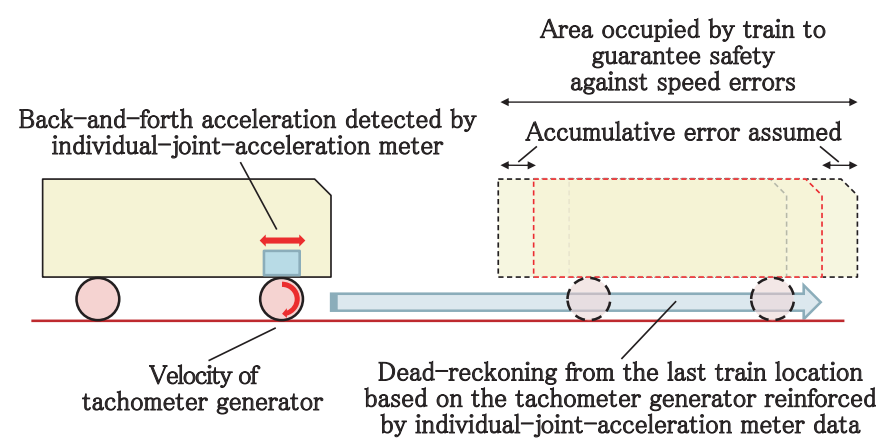

Fig. 17 Train location detection by dead-reckoning

\section{Initial position detection method}

The initial position detection method which detects location before the turnout direction is determined is described below.

\subsection{Usual initial position detection}

Initial position detection is performed at the time of the entry to a main track from a depot, storage track, etc. Since normal operations are not performed in depots or storage tracks, etc., they are not treated in the same way as in the controlled area, and these areas are defined as non-controlled. Since connection from non-controlled areas to the main track is usually via a single line in the case of secondary lines, a control boundary is set on the track (Fig. 18).

By completing the following procedures the on board controller determines that the train has reached the control boundary of a non-controlled area and the location defined as the control boundary is set as the initial position of the train. However, the premise is that GPS and MSAS are operable within a specified belt area inside the non-controlled area leading up to the aforementioned boundary.

(a) After the rolling stock power supply is switched on and the on board controller is functioning, permission to enter into operation should be received from the ground equipment

(b) When HPL satisfies the accuracy requirement in terms of train location detection, the GPS positioning results when jointly used with MSAS should overlap with the non-controlled area

(c) As the train moves the GPS positioning results when jointly used with MSAS should reach within a pre-

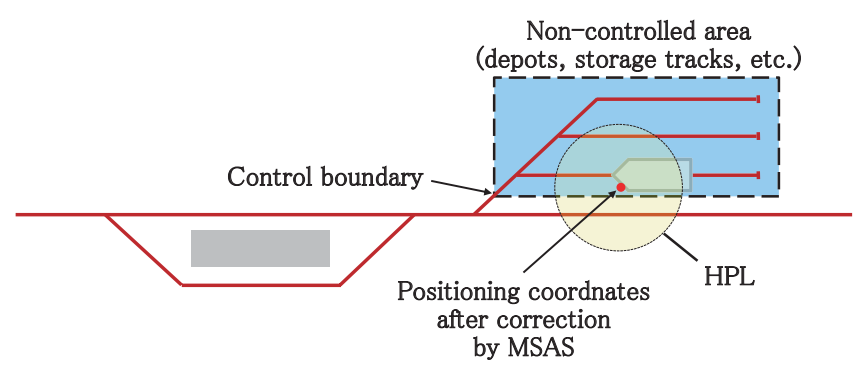

Fig. 18 Non-controlled area and control boundary determined range of the control boundary location According to the above procedure, before the ground equipment issues permission to enter into operation, the location of the train set can be confirmed by cross checking it with the departure information as it leaves the depot, etc. In addition, the overlap of the GPS positioning results when used together with MSAS and the noncontrolled area can be checked. Furthermore, GPS positioning results while moving towards the control boundary can be checked continuously using speed and direction data from the tachometer generator which is backed up by the individual-joint-acceleration meter, as well as ground speed and direction obtained from the GPS. The initial position is set at the control boundary when a train it is within the pre-determined distance range of the control boundary only when a train leave a non-controlled area to engage on the main line via a single track. In addition, initial position detection, rolling stock direction and driving direction can also be determined by conditions such as: cab selection switches, the reverser, notch, or the installation orientation of the individual-joint-acceleration meter. If the on board controller transmits an initial position to the ground equipment, the ground equipment will start tracking the train and transmit stop limits and route information to the on board controller. Once the on board controller has received this, it shifts to a normal location and speed detection mode.

If we follow this procedure, even though two or more locations where initial position detection are performed are located in the line, there will be no problem, if these locations are separate more than the pre-determined distance required by the accuracy of train location detection. When a train stops on a main line, the ground equipment should memorise the train position just before the rolling stock is powered off. This position should then be transmitted back to the on board controller immediately after the train is powered on again.

When a line is only partially covered by this system, a process similar to initial position detection must be performed when a train moves between a non-installed zone and an installed zone. This process is applied on a single line basis, like in the method mentioned above, but differs since it is performed during normal operations. In order to ensure that the system is adapted to high speed operation, matters such as setting a broad non-controlled area and ensuring a suitable belt area leading up to the control boundary where checks are performed for initial position determination, should be taken into account. In addition, in order prevent entry of the train if the initial position detection is not complete, a link must be made with the operational safety system of the non-installed zone.

Since after initial position detection, running is limited to a single track by virtue of the block section and route information transmitted together with a stop limits from the ground equipment, the on board controller performs location correction and dead-reckoning on the track. If the stop limit of a turnout is exceeded the train location becomes undefined. 


\subsection{Recovery from undefined location mode}

Normal train control becomes impossible once location is undefined within the control range of the system, and so location must recovered as quickly as possible. This is done via fixed location information and other back up systems on the track as mentioned above. However, there are locations on the main track where there are two or more tracks laid, or where communication is impossible. As shown in Fig. 1, this train control system functions on a single track basis, and since radio communication zones are set up to cover at least the area of a station yard, the following may be done:

(a) Station and surrounding area (radio communication is possible)

After the re-booting the on board controller, it receives train location, stop limit and route information from the ground equipment just before the train location becomes undefined. This information can be used for pinpointing a place on the track and as back up. Since the train may have moved up to the stop limit, recovery happens as location correction is performed.

(b) Between stations (one line, radio communication is impossible)

Recovery is achieved using the method for initial position detection in the case of movement between a non-installed zone to an installed zone. After re-booting the on board controller, it checks GPS positioning results when used together with MSAS, while moving through a location where GPS and MSAS are operable between stations, and the system recovers when stop limit and route information from ground equipment is received, before reaching the following station.

If ground equipment or wireless installations are out of order when location becomes undefined, this back up information cannot be provided. A system solution could be used to overcome such cases, but given the complexity and expense involved in doing so, human intervention is probably the best solution.

\section{Issues when using MSAS}

As described in Section 4.2, in order to use MSAS as a basis for a train safety control system, the user must fully comprehend how it functions and understand its operational performance. The SBAS receiver certified for use in aviation is likely to be very expensive. The problem of the high price of receivers can be avoided by using an uncertified receiver based on the standard (technical standard of a SBAS receiver, MOPS RCTA-DO-229C) used for these measurements. However, MSAS functions such as protection level, must be verified to ensure they are equivalent to those of certified instruments.

\section{Conclusions}

In order to lower the cost of on board location and speed detection functions in a radio communication train control system used for secondary lines, studies were carried out and applicability investigated for the performance of GPS positioning when used in combination with MSAS. Based on results obtained from tests carried out on Railway Technical Research Institute test tracks it was shown that there was a possibility of locating the position of the vehicle on the track by using HPL when running under open sky. The conclusion therefore is that a system equipped only with on board functions, and which did not use ground installations, can be built.

\section{References}

[1] Sakai et al., "Improvement of performance on MSAS," Electronic Navigation Research Institute research presentation meeting lecture outline, Vol.8, June, 2008, (in Japanese).

[2] Yamamoto, H., Nishibori, N., "CARAT," RRR, Vol. 56, No. 9, pp. 8-9, 1999 (in Japanese). 